\title{
Influência do exercício físico nas alterações cardiovasculares induzidas pelo hipotireoidismo
}

\section{Influence of physical exercise on cardiovascu- lar changes induced by hypothyroidism}

Alesandra Araújo de Souza ${ }^{1,2}$

Aluísio Henrique Rodrigues de Andrade Lima²

Thereza Sarmento da Nóbrega ${ }^{1}$

Alexandre Sérgio Silva ${ }^{1}$

\section{Resumo}

O hipotireoidismo é uma disfunção da tireóide que não produz seus hormônios ou só o faz através de uma alta produção do hormônio tireoestimulante. Essa enfermidade está associada ao desenvolvimento de disfunções cardiovasculares diversas. Esta revisão traz à comunidade científica e aos profissionais da saúde, informações atualizadas sobre as repercussões cardiovasculares do hipotireoidismo, sua influência na capacidade para o exercício físico, e o potencial do exercício físico para minimizar ou restaurar estas disfunções. Um levantamento dos estudos publicados entre 1995 e 2011 foi conduzido na base de dados PubMed utilizando os descritores: physical exercise, blood pressure, cardiovascular, vascular endothelial, oxidative stress, autonomic nervous system, cardiac function, subclinical hypothyroidism e hypothyroidism. Foram considerados, os artigos que avaliaram a função cardiovascular de hipotireoideos subclínicos e clínicos, e a influência do exercício físico sobre as funções cardiovasculares afetadas nesta doença. Verificamos que a capacidade aeróbia de hipotireoideos subclínicos e clínicos está reduzida, e que a função endotelial, o estresse oxidativo e a função autonômica estão prejudicados nestes sujeitos. O exercício físico parece ser capaz de restaurar a função endotelial, apesar de não fazer o mesmo na disfunção autonômica. Não existem investigações associando o efeito do exercício físico na função cardíaca e no estresse oxidativo. Apesar de ser uma enfermidade tão prevalente quanto o diabetes, não existe uma recomendação para a prescrição de exercício físico para os hipotireoideos, tal como há para obesos, cardiopatas e diabéticos. Perspectivas sobre como avançar no conhecimento da prescrição do exercício físico são apresentadas.

\section{Palavras-chave}

Hipotireoidismo; Exercício físico; Sistema cardiovascular.

\begin{abstract}
Hypothyroidism is a disorder of the thyroid gland that does not produce its hormones or only does in response to a high production of thyroid-stimulating hormone. This disease is associated with the development of various cardiometabolic disorders. This review brings to the scientific community and health professionals, updated information about the cardiovascular effects of hypothyroidism, its influence on physical exercise capacity, and the potential of physical exercise to minimize or restore the dysfunction induced by hypothyroidism. A survey of studies published between 1995 to 2011 was conducted in PubMed using the keywords: physical exercise, blood pressure, cardiovascular, vascular endothelial, oxidative stress, autonomic nervous system, cardiac function, subclinical hypothyroidism and hypothyroidism. We considered articles that evaluated cardiovascular function of subclinical and clinical hypothyroidism, and the influence of physical exercise on cardiovascular dysfunction induced by this disease. We found that the aerobic capacity of subclinical and clinical hypothyroid patients is reduced, and endothelial function, oxidative stress and autonomic function are impaired in these subjects. Physical exercise seems to be able to restore endothelial function, although not autonomic dysfunction. There are no investigations involving the effect of physical exercise on cardiac function and oxidative stress. Despite being a disease as prevalent as diabetes, there is not a recommendation for physical exercise prescription for hypothyroidism as there is for obesity, heart disease and diabetes. Perspectives on how to advance in the knowledge of physical exercise prescription is presented.
\end{abstract}

\section{Keywords}

Hypothyroidism; Physical exercise; Cardiovascular system.
Rev Bras Ativ Fis Saúde p. 370-382 DOI: http://dx.doi.org/10.12820/2317. 1634.2012v17n5p370

1 Laboratório de Estudos do Treinamento Físico Aplicado ao Desempenho e à Saúde, Universidade Federal da Paraíba, João Pessoa, PB, Brasil

2 Programa Associado de Pós-graduação em Educação Física Universidade de Pernambuco/Universidade da Paraíba, Recife, $P E$, Brasil. 


\section{INTRODUÇÃO}

O hipotireoidismo é uma doença metabólica caracterizada por uma disfunção da glândula tireóide. Sujeitos com disfunção nesta glândula são chamados de hipotireoideos. Suas glândulas não conseguem produzir os hormônios tiroxina (T4) e triiodotironina (T3) ou só o fazem à custa de elevadas secreções do hormônio tireoestimulante (TSH). ${ }^{(1)}$ Esta doença se manifesta com caráter subclínico, quando ocorre produção normal de T3 (de 9,1 a 25,6 pmol/1) e T4 (de 3,20 a 9,2 pmol/1), mas apenas à custa da alta produção do TSH, que apresenta valores maiores do que 4,5mIU/L. ${ }^{(2-3)}$ A outra forma de manifestação é o hipotireoidismo clínico, no qual a concentração de TSH está elevada, mas mesmo assim os níveis de T3 e T4 permanecem baixos. A prevalência de hipotireoidismo subclínico no mundo é de 4 a 10\%, sendo que de 3 a 18\% desses casos evoluem para a forma clínica. ${ }^{(2)}$

Além das repercussões clássicas no metabolismo, o hipotireoidismo está relacionado também com disfunções cardiovasculares, que podem ser explicadas pelo fato de haver receptores dos hormônios tireoidianos nas células miocárdicas e endoteliais..$^{(1,4)}$ As disfunções cardiovasculares já são observadas no hipotireoidismo subclínico ${ }^{(2)}$, pois, uma produção inadequada de TSH tem sido associada com maiores valores de pressão arterial sistólica e diastólica ${ }^{(6)}$, disfunção endotelial ${ }^{(7-8)}$, estresse oxidativo $^{(9)}$ e desbalanço simpatovagal. ${ }^{(10-12)}$

O exercício físico tem sido indicado como tratamento não farmacológico para doenças crônico-degenerativas como a hipertensão arterial, o diabetes e a obesidade. ${ }^{(13-15)}$ Além de ser capaz de reduzir pressão arterial, a glicemia em jejum e a gordura corporal, vários estudos demonstram que o exercício físico melhora a função endotelial, a resistência vascular periférica e o balanço simpatovagal de hipertensos, diabéticos e obesos. ${ }^{(16-17)}$ No entanto, estes benefícios não podem ser extrapolados diretamente para os hipotireóideos pelo fato de que as disfunções cardiometabólicas provocadas por esta doença têm como fator associado a presença de receptores de T3 e T4 nas células cardíacas e vasculares.

Portanto, esta revisão foi conduzida com a finalidade de trazer, à comunidade científica e aos profissionais da área de saúde, informações sobre os efeitos deletérios do hipotireoidismo na função miocárdica, resistência vascular periférica, função endotelial, estresse oxidativo e atividade nervosa autonômica. Em seguida, são apresentados o papel do exercício físico na minimização dos efeitos do hipotireoidismo subclínico ou clínico nas funções cardiovasculares e as perspectivas acerca da prescrição de exercícios físicos para hipotireoideos.

\section{MÉTODOS}

A busca por artigos foi realizada na base de dados PubMed entre o período de 1995 a 2011. Para realização da busca adotou-se o cruzamento dos seguintes termos em inglês: 1 - physical exercise; 2 - blood pressure; 3 - cardiovascular; 3 - vascular endothelial; 4- oxidative stress; 5 - autonomic nervous system; 6 - cardiac function; 7 - subclinical hypothyroidism; 8 - hypothyroidism.

Após a combinação dos descritores, recorreu-se à leitura dos títulos e resumos a fim de se determinar quais artigos atendiam aos critérios de inclusão, sendo consideradas as investigações conduzidas com hipotireoideos subclínicos ou clínicos e que tivessem avaliado as respostas cardiovasculares ao exercício físico. Foram excluídos os manuscritos que avaliaram a influência do exercício físico na 
força muscular, equilíbrio ou agilidade de hipotireoideos clínicos ou subclínicos; que tenham utilizado voluntários com hipotireoidismo congênito, câncer no eixo hipófise-tireóide ou tireoidectomizados, e revisões sistemáticas ou meta-análises. Todos os artigos foram avaliados independentemente por dois pesquisadores, e, em caso de divergências, um terceiro integrante do estudo decidiu pela inclusão ou não do manuscrito.

\section{RESULTADOS}

Na tabela 1 estão apresentadas as características autonômicas, hemodinâmicas e cardiovasculares de indivíduos com hipotireoidismo subclínico e clínico relatadas em 12 estudos selecionados. Para caracterizar o estado destas funções cardiovasculares nos hipotireoideos, os autores as compararam com sujeitos eutireoideos. Estes estudos mostram piora da função cardíaca, do estado oxidativo e da atividade nervosa autonômica em hipotireoideos. Por outro lado, quando a reposição de levotiroxina foi adotada ocorreu restauração parcial ou comparável aos eutireoideos nestas variáveis.

$\mathrm{Na}$ tabela 2 estão apresentados os dados de oito estudos que analisaram a influência do exercício físico sobre a função cardíaca, variáveis hemodinâmicas e capacidade para o exercício físico de hipotireoideos subclínicos e clínicos. Nota-se que existem poucos estudos, e que a maior parte deles não adotou sessões de treinamento físico, mas sim testes incrementais para avaliar as respostas cardiovasculares pretendidas. $\mathrm{O}$ único que adotou o treinamento físico utilizou como protocolo de intensidade moderada.

\section{DISCUSSÃO}

\section{Potenciais contribuições do exercício físico nas disfunções cardio- vasculares induzidas pelo hipotireoidismo}

\section{- Função cardíaca}

O sistema cardiovascular é um dos principais alvos da ação dos hormônios tireoidianos. ${ }^{(33)}$ Devido à presença de receptores específicos de T3 nas células cardíacas e vasculares ${ }^{(34)}$, alterações promovidas pela redução da produção dos hormônios tireoidianos implicam em diminuição do débito cardíaco, aumento da resistência vascular periférica, disfunção sistólica e diastólica. Isso ocorre, em grande parte, devido à contratilidade miocárdica comprometida. ${ }^{(35)}$ Os hormônios tireoidianos regulam a expressão gênica da cadeia $\alpha$ e $\beta$ da miosina, que são responsáveis pelo mecanismo de contração muscular. ${ }^{(36)} \mathrm{A}$ expressão positiva da cadeia $\alpha$ da miosina através do hormônio tireoidiano é capaz de promover maior contratilidade miocárdica comparada com a cadeia $\beta$. Porém, em estado hipofuncionante da tireóide não há expressão positiva da cadeia $\alpha$ da miosina. Nesta condição, o miocárdio é menos eficiente para contrair e manter o volume de ejeção necessário para o exercício físico. ${ }^{(36)}$ Estas mudanças são menos evidentes no repouso e mais proeminentes durante o exercício físico.

De fato, Casimiro-Lopes et al. ${ }^{(27)}$ verificaram que em ratos hipotireoideos clínicos submetidos a sessões de natação, o tempo de exercício físico se apresentou reduzido ao longo de diferentes sessões de exercício físico. Ainda que hipotireoideos subclínicos apresentem níveis normais dos hormônios tireoideanos, a contratilidade miocárdica já não se encontra preservada. De fato, Brenta et al. ${ }^{(30)}$ compararam 
Tabela 1 - Condições hemodinâmicas e cardiovasculares apresentadas pelos hipotireoideos subclínicos e clínicos e respostas ao tratamento com levotiroxina.

\begin{tabular}{|c|c|c|c|c|c|c|}
\hline Estudo & $\begin{array}{c}\mathrm{N} \\
\text { idade }\end{array}$ & $\begin{array}{l}\text { Tipo de } \\
\text { Hipotireoi- } \\
\text { dismo }\end{array}$ & Tipo de Amostra & Objetivo/Tratamento & $\begin{array}{l}\text { Condição em } \\
\text { relação aos } \\
\text { eutireoideos }\end{array}$ & $\begin{array}{l}\text { Tratamento com } \\
\text { levotiroxina }\end{array}$ \\
\hline $\begin{array}{l}\text { Kisso et } \\
\text { al.(18) }\end{array}$ & $\begin{array}{l}-/-- \\
---\end{array}$ & $\mathrm{HC}$ & Modelo Animal & $\begin{array}{c}\text { Efeito da função tireoidea reduzida na } \\
\text { estrutura e função cardíaca }\end{array}$ & --- & $\begin{array}{l}\downarrow R P A \\
\downarrow F E\end{array}$ \\
\hline $\begin{array}{l}\text { Coria et } \\
\text { al.(9) }\end{array}$ & $\begin{array}{c}81 F /-- \\
---\end{array}$ & $\begin{array}{l}\mathrm{HSC} \\
\mathrm{HC}\end{array}$ & Humanos & $\begin{array}{l}\text { Caracterização do estado oxidativo de } \\
\text { mulheres hipotireoideas subclínicas e } \\
\text { clínicas/ Não houve tratamento. }\end{array}$ & $\begin{aligned} \text { HSC: } & \leftrightarrow \text { TBARS } \\
& \uparrow N O \\
\leftrightarrow & \text { PON }-1 \\
\text { HC: } & \leftrightarrow \text { TBARS } \\
& \uparrow N O \\
\leftrightarrow & \text { PON }-1\end{aligned}$ & --- \\
\hline $\begin{array}{l}\text { Dieckman } \\
\text { et al. (19) }\end{array}$ & $\begin{array}{l}8 \mathrm{~F} / 3 \mathrm{M} \\
47 \text { anos }\end{array}$ & $\mathrm{HC}$ & Humanos & $\begin{array}{l}\text { Relação entre mudança da RVP e } \\
\text { hormônios não tireoideos de } \mathrm{HC} / \text { Esta- } \\
\text { belecimento de estado eutireoideo }\end{array}$ & $\begin{array}{l}\uparrow \mathrm{RVP} \\
\uparrow \mathrm{PAS} \\
\leftrightarrow \mathrm{PAD} \\
\leftrightarrow \mathrm{ET}-1\end{array}$ & $\begin{array}{l}\downarrow R V P \\
\downarrow P A S \\
\leftrightarrow \text { PAD } \\
\leftrightarrow \text { ET-1 }\end{array}$ \\
\hline $\begin{array}{l}\text { Gauna et al. } \\
(20)\end{array}$ & 18 & $\begin{array}{l}\mathrm{HSC} \\
\mathrm{HC}\end{array}$ & Humanos & $\begin{array}{l}\text { Comparar a FVE entre HSC, HC e euti- } \\
\text { reoideos/ Não houve tratamento. }\end{array}$ & $\begin{array}{l}\text { HSC: } \downarrow F V E \\
\text { HC: } \downarrow \text { FVE }\end{array}$ & --- \\
\hline $\begin{array}{l}\text { Giannat- } \\
\text { tasio et al. } \\
(21)\end{array}$ & $\begin{array}{c}9 \mathrm{~F} / 2 \mathrm{M} \\
59 \pm 2,3 \text { anos }\end{array}$ & $\mathrm{HC}$ & Humanos & $\begin{array}{l}\text { Investigar a CA de } \mathrm{HC} \text { / Terapia de } \\
\text { reposição hormonal }\end{array}$ & $\begin{aligned} \leftrightarrow & \text { PAS } \\
\leftrightarrow & \text { PAD } \\
& \downarrow \text { CA }\end{aligned}$ & $\begin{array}{l}\text { PAS } \\
\text { PAD } \\
\text { CA }\end{array}$ \\
\hline $\begin{array}{l}\text { Tadei et } \\
\text { al.(22) }\end{array}$ & $\begin{array}{l}12 \mathrm{~F} / 2 \mathrm{M} \\
39,6 \pm 8,4 \\
\text { anos }\end{array}$ & HSC & Humanos & $\begin{array}{l}\text { Relação entre reatividade vascular e } \\
\text { HSC/ Terapia de reposição hormonal }\end{array}$ & $\begin{array}{l}\leftrightarrow \mathrm{PAS} \\
\leftrightarrow \mathrm{PAD} \\
\leftrightarrow \mathrm{FSA}\end{array}$ & $\begin{aligned} \leftrightarrow & \text { PAS } \\
\leftrightarrow & \text { PAD } \\
& \uparrow F S A\end{aligned}$ \\
\hline $\begin{array}{l}\text { Moulakakis } \\
\text { et al. (23) }\end{array}$ & 20 & $\mathrm{HC}$ & Modelo Animal & Avaliar estado oxidativo na aorta de $\mathrm{HC}$ & --- & $\begin{array}{c}\uparrow 8 \text {-oxo-dG } \\
\uparrow M D A\end{array}$ \\
\hline $\begin{array}{l}\text { Torun et } \\
\text { al.(24) }\end{array}$ & $\begin{array}{l}60 \\
---\end{array}$ & $\begin{array}{l}\mathrm{HSC} \\
\mathrm{HC}\end{array}$ & Humanos & $\begin{array}{l}\text { Influência do HSC e HC na concentra- } \\
\text { ção sérica de MDA e ENO/ Não houve } \\
\text { tratamento }\end{array}$ & $\begin{array}{c}\text { HSC: } \leftrightarrow \text { EANT } \\
\uparrow M D A \\
\text { HC: } \leftrightarrow \text { EANT } \\
\uparrow M D A\end{array}$ & --- \\
\hline $\begin{array}{l}\text { Galetta et } \\
\text { al. (11) }\end{array}$ & $\begin{array}{l}29 \mathrm{~F} / 13 \mathrm{M} \\
53,2 \pm 14,2 \\
\text { anos }\end{array}$ & $\mathrm{HSC}$ & Humanos & $\begin{array}{l}\text { Avaliação da dispersão do segmento } \\
\text { QT em HSC/Terapia de reposição } \\
\text { hormonal com levotiroxina }\end{array}$ & $\begin{array}{l}\uparrow D I Q T \\
\downarrow V F C\end{array}$ & $\begin{array}{l}\downarrow D I Q T \\
\uparrow V F C\end{array}$ \\
\hline $\begin{array}{l}\text { Sahin et al. } \\
\text { (12) }\end{array}$ & 31 & HSC & Humanos & $\begin{array}{l}\text { Avaliar o efeito do HSC no balanço } \\
\text { simpatovagal/ Não houve tratamento }\end{array}$ & $\begin{aligned} & \leftrightarrow \text { RR } \\
\leftrightarrow & \text { SDNN } \\
\leftrightarrow & \text { rMSSD }\end{aligned}$ & --- \\
\hline $\begin{array}{l}\text { Celik et } \\
\text { al.(25) }\end{array}$ & $\begin{array}{c}36 \mathrm{~F} / 4 \mathrm{M} \\
48 \pm 13 \text { anos }\end{array}$ & $\mathrm{HC}$ & Humanos & $\begin{array}{c}\text { Avaliação da função autonômica } \\
\text { cardíaca de hipotireoideos/ Reposição } \\
\text { com levotiroxina }\end{array}$ & $\begin{array}{c}\leftrightarrow \mathrm{RR} \\
\downarrow \mathrm{SDNN} \\
\leftrightarrow \mathrm{rMSSD}\end{array}$ & $\begin{aligned} & \leftrightarrow \text { RR } \\
\leftrightarrow & \text { SDNN } \\
\leftrightarrow & \text { rMSSD }\end{aligned}$ \\
\hline $\begin{array}{l}\text { Cacciatori } \\
\text { et al.(26) }\end{array}$ & $\begin{array}{l}70 \mathrm{~F} \\
52,1 \pm 5,3 \\
\text { anos }\end{array}$ & $\mathrm{HC}$ & Humanos & $\begin{array}{c}\text { Avaliação da função autonômica } \\
\text { cardíaca/ Estabelecimento de estado } \\
\text { eutireoideo }\end{array}$ & 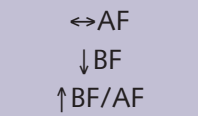 & $\begin{aligned} & \leftrightarrow \mathrm{AF} \\
& \leftrightarrow \mathrm{BF} \\
& \leftrightarrow \mathrm{BF} / \mathrm{AF}\end{aligned}$ \\
\hline
\end{tabular}

F=gênero feminino; $M=$ gênero masculino; $\uparrow$ = aumento; $\downarrow=$ diminuição; $\leftrightarrow$ = sem mudança; HSC= hipotireoidismo subclínico; HC= hipotireoidismo clínico; TBARS= substâncias reativas ao ácido tiobarbitúrico; NO= óxido nítrico; $P O N-1=$ paraoxonase; $D I Q T=$ dispersão do intervalo $\mathrm{QT} ; \mathrm{VFC}=$ variabilidade da frequência cardíaca; $\mathrm{RVP}=$ resistência vascular periférica; $\mathrm{PAS}=$ pressão arterial sistólica; $P A D=$ pressão arterial diastólica; $\mathrm{ET}-1=$ endotelina-1; $\mathrm{FVE}=$ função ventricular esquerda; $\mathrm{RPA}=$ rigidez da parede arterial; $\mathrm{FE}=$ fração de ejeção; $\mathrm{FC}=$ frequência cardíaca; $C A=$ complacência arterial; $F S A=$ fluxo sanguíneo do antebraço; 8-oxo-dG= derivado oxidado da deoxiguanosina; $M D A=$ malondialdeído; DIQT= dispersão do segmento QT; EANT= estado antioxidante; RR= intervalo RR; $D S N N=$ desvio padrão de todos os intervalos RR;

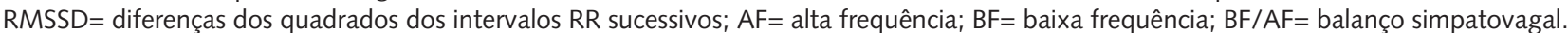

a função ventricular esquerda de hipotireoideos subclínicos e voluntários saudáveis durante um protocolo incremental na bicicleta ergométrica e observaram que não houve diferença estatística para a fração de ejeção ventricular esquerda de repouso entre os grupos. No entanto, durante o exercício físico os voluntários com hipotireoidismo subclínico possuíam fração de ejeção ventricular significativamente menor do que o grupo controle. 
Tabela 2 - Respostas hemodinâmicas e cardiovasculares de hipotireoideos subclínicos após intervenção com testes incrementais ou treinamento com exercício aeróbio.

\begin{tabular}{|c|c|c|c|c|c|c|c|c|c|}
\hline Estudo & $N(F / M)$ & $\begin{array}{l}\text { Tipo de } \\
\text { Hipoti- } \\
\text { reoidismo }\end{array}$ & $\begin{array}{l}\text { Tipo de } \\
\text { Amostra }\end{array}$ & $\begin{array}{l}\text { Tipo de } \\
\text { Exercício }\end{array}$ & Intensidade & $\begin{array}{c}\text { Duração } \\
\text { da sessão } \\
\text { (min) }\end{array}$ & $\begin{array}{c}\text { Frequência } \\
\text { Semanal } \\
\text { (dias/semana) }\end{array}$ & $\begin{array}{l}\text { Duração do } \\
\text { Treinamento } \\
\text { (semanas) }\end{array}$ & Resultados \\
\hline $\begin{array}{l}\text { Casimiro- } \\
\text { Lopes et } \\
\text { al.(27) }\end{array}$ & $--/--$ & $\mathrm{HC}$ & $\begin{array}{l}\text { Modelo } \\
\text { Animal }\end{array}$ & $\begin{array}{l}\text { Teste máximo } \\
\text { na natação }\end{array}$ & $\begin{array}{c}5 \% \text { do peso } \\
\text { corporal }\end{array}$ & --- & --- & --- & $\begin{array}{c}\downarrow T M E \\
\uparrow G \text { lic.M } \\
\uparrow \text { Lept. } \\
\downarrow \text { Cortic. }\end{array}$ \\
\hline $\begin{array}{l}\text { McAllister et } \\
\text { al.(28) }\end{array}$ & --- & $\mathrm{HC}$ & $\begin{array}{l}\text { Modelo } \\
\text { Animal }\end{array}$ & Teste aeróbio & $30 \mathrm{~m} / \mathrm{min}$ & 60 & 5 & $10-15$ & $\downarrow F S$ \\
\hline $\begin{array}{l}\text { Bausch et } \\
\text { al.(29) }\end{array}$ & --- & $\mathrm{HC}$ & $\begin{array}{l}\text { Modelo } \\
\text { Animal }\end{array}$ & $\begin{array}{l}\text { Contrações } \\
\text { Tetânicas }\end{array}$ & $30 / \mathrm{min}$ & --- & --- & --- & $\uparrow F S$ \\
\hline $\begin{array}{l}\text { Brenta et } \\
\text { al.(30) }\end{array}$ & $9 F / 1 M$ & HSC & Humanos & $\begin{array}{c}\text { Exercício } \\
\text { Incremental }\end{array}$ & $\begin{array}{c}150 \mathrm{Kpm} \text { a } \\
\text { cada } 2 \mathrm{~min} 30 \mathrm{~s}\end{array}$ & --- & --- & --- & $\begin{array}{l}\downarrow \text { FVES } \\
\downarrow \text { FVED }\end{array}$ \\
\hline $\begin{array}{l}\text { Mainenti et } \\
\text { al.(31) }\end{array}$ & 15F/-- & HSC & Humanos & $\begin{array}{l}\text { Teste Ergoes- } \\
\text { pirométrico }\end{array}$ & $\begin{array}{l}\text { Aumento de } \\
3 \% \text { de inclina- } \\
\text { ção a cada } 2 \\
\text { minutos }\end{array}$ & $\begin{array}{l}\text { Até a } \\
\text { fadiga }\end{array}$ & --- & --- & $\begin{array}{l}\leftrightarrow \text { PASpico } \\
\leftrightarrow \text { PADpico } \\
\downarrow \text { PASRec.1 } \\
\leftrightarrow \text { PASRec.3 } \\
\leftrightarrow \text { PADRec.1 } \\
\leftrightarrow \text { PADRec.3 }\end{array}$ \\
\hline $\begin{array}{l}\text { Arem et } \\
\text { al.(32) }\end{array}$ & $6 F / 2 M$ & HSC & Humanos & $\begin{array}{c}\text { Exercício } \\
\text { Incremental }\end{array}$ & $70 \%$ da FCM & $\begin{array}{l}\text { Até a } \\
\text { fadiga }\end{array}$ & --- & --- & $\leftrightarrow$ FEExerc. \\
\hline $\begin{array}{l}\text { Akcakoyun et } \\
\text { al.(10) }\end{array}$ & $\begin{array}{c}14 \mathrm{~F} / 11 \mathrm{M} \\
36 \pm 10 \\
\text { anos }\end{array}$ & HSC & Humanos & $\begin{array}{l}\text { Teste na } \\
\text { esteira }\end{array}$ & $\begin{array}{c}11,5 \pm 2,2 \\
\text { METs }\end{array}$ & --- & --- & --- & $\begin{aligned} \leftrightarrow & \text { FCRec. } \\
& \leftrightarrow I C\end{aligned}$ \\
\hline $\begin{array}{l}\text { Xiang et al. } \\
\text { (3) }\end{array}$ & 30F/-- & HSC & Humanos & $\begin{array}{c}\text { Caminhada } \\
\text { Jogo }\end{array}$ & $\begin{array}{c}60 \% \text { da FCM } \\
70 \%-75 \% \text { da } \\
\text { FCM }\end{array}$ & $\begin{array}{l}25-30 \\
40-45\end{array}$ & $\begin{array}{l}3-4 \\
4-6\end{array}$ & 24 & $\begin{array}{c}\uparrow D M F \\
\downarrow C T \\
\uparrow H D L \\
\downarrow L D L \\
\downarrow T G \\
\downarrow P C R\end{array}$ \\
\hline
\end{tabular}

F=gênero feminino; $M=$ gênero masculino; $\uparrow$ = aumento; $\downarrow=$ diminuição; $\leftrightarrow$ = sem mudança; HSC= hipotireoidismo subclínico; HC= hipotireoidismo clínico; $\mathrm{FCM}=$ frequência cardíaca máxima; $\mathrm{DMF}=$ dilatação mediada pelo fluxo; $\mathrm{CT}=$ colesterol total; $\mathrm{HDL}=$ lipoproteínas de alta densidade; $\mathrm{LDL}=$ lipoproteínas de baixa densidade; $\mathrm{TG}=$ triglicerídeos; $\mathrm{PCR}=$ proteína $\mathrm{C}$-reativa; $\mathrm{TME}=$ tempo máximo de exercício; Glic. Musc. = glicogênio muscular; Lept. = leptina; Cortic. = corticosterona; FVES= função ventricular esquerda sistólica; FVED= função ventricular esquerda diastólica; PASpico= pressão arterial sistólica no pico do exercício; PADpico= pressão arterial diastólica no pico do exercício; PASRec.1= pressão arterial sistólica após 1 minutos de recuperação; PASRec.3= pressão arterial sistólica após 3 minutos de recuperação; FERep.= fração de ejeção no repouso; FEExerc.= fração de ejeção no exercício; FS= fluxo sanguíneo; $\mathrm{m} / \mathrm{min}=$ metros/minuto.

Apesar de Brenta et al. ${ }^{(30)}$ não haverem investigado se essa pior fração de ejeção ventricular comprometeu a capacidade para o exercício físico, Mainenti et al. ${ }^{(37)}$ avaliaram as manifestações do hipotireoidismo subclínico na resposta cardiopulmonar durante o exercício físico incremental na esteira ergométrica. As pacientes obtiveram valores menores no pico do exercício físico para fração expirada de $\mathrm{O}_{2}$, na duração do exercício físico e na carga máxima do teste comparado com indivíduos saudáveis. Segundo os autores, a fadiga precoce pode ser explicada pela intolerância à acidose metabólica proveniente do aumento da contribuição do metabolismo anaeróbio durante o esforço, ou mesmo à menor capacidade do organismo hipotireoideo subclínico de metabolizar o lactato produzido no exercício físico. Para além do prejuízo na contratilidade miocárdica, o hipotireoidismo subclínico induz alterações no sistema cardiovascular em repouso, incluindo diminuição do fluxo sanguíneo e aumento da resistência periférica total. ${ }^{(18-19)}$

Tang et al. ${ }^{(38)}$ avaliaram a insuficiência cardíaca em ratos com hipotireoidismo subclínico induzido por meio da administração de $0,025 \%$ de propiltiouracil ao 
longo de 6 meses. Os resultados demonstraram que ainda no estágio subclínico, o hipotireoidismo já produz alterações semelhantes à insuficiência cardíaca, tais como aumento da cavidade do ventrículo esquerdo associado à diminuição da massa cardíaca, diminuição do débito cardíaco e disfunção sistólica progressiva. Resultados semelhantes foram encontrados em humanos por Niafar et al. ${ }^{(39)}$, que estudaram 35 mulheres hipotireoideas subclínicas com média de 40 anos de idade. A frequência de disfunção diastólica ventricular esquerda foi significativamente maior nas hipotireoideas subclínicas quando comparadas com o grupo controle. Corroborando com esses dados, Gauna et al. ${ }^{(20)}$ verificaram que a função ventricular esquerda esteve comprometida de forma semelhante tanto em hipotireoideos subclínicos quanto clínicos quando comparados com um grupo controle.

Apesar deste razoável nível de evidências, pelo menos com base nos critérios adotados na metodologia para a busca dos artigos, não encontramos nenhuma investigação que tivesse avaliado a influência do exercício físico na função miocárdica de pacientes com hipotireoidismo subclinico ou clínico.

\section{- Resistência vascular periférica}

O fluxo sanguíneo e a vasodilatação se apresentam reduzidos em pacientes com hipotireoidismo subclínico em uma relação dose-dependente com os níveis séricos de TSH. ${ }^{(8)}$ Existem evidências de que ao haver acoplamento dos hormônios tireoideanos nas células endoteliais há liberação de substâncias vasoconstritoras como a endotelina- $1^{(40)}$, e vasodilatadoras como a adrenomedulina ${ }^{(41)}$ e óxido nítrico. ${ }^{(42)}$

Em estudo com 65 indivíduos com hipotireoidismo clínico foi observado que a concentração de TSH esteve positivamente correlacionada com a rigidez arterial. ${ }^{(7)}$ Como observado por Napoli et al. ${ }^{(43)}$, pacientes hipotireoideos subclínicos e clínicos apresentaram vasodilatação prejudicada. Contudo, esse efeito foi mais pronunciado nos hipotireoideos clínicos. Desse modo, os autores sugerem que esse efeito não se deve tão somente às concentrações de TSH, mas também à deficiência dos hormônios tireoidianos.

A resistência vascular periférica pode estar comprometida devido à estrutura da parede arterial. Em pacientes com hipotireoidismo clínico, Giannattasio et al. ${ }^{(21)}$, observaram que a parede da artéria radial tinha maior espessura comparada aos indivíduos saudáveis, mas após terapia de reposição hormonal houve redução desta rigidez nos hipotireoideos.

Embora o efeito do exercício físico sobre a função vasodilatadora venha sendo bem investigado entre hipertensos, poucos estudos avaliaram se pacientes hipotireoideos responderiam ao exercício físico com a mesma melhora na capacidade vasodilatadora observada entre hipertensos. McAllister et al. ${ }^{(28)}$ demonstraram que o fluxo sanguíneo prejudicado no repouso se mantinha mesmo no exercício físico em ratos com hipotireoidismo clínico simulado. Por outro lado, Bausch et al. ${ }^{(29)}$ verificaram que após contrações tetânicas, com duração de dois a três segundos, houve restauração do fluxo sanguíneo das artérias de pequenos grupos musculares em ratos com hipotireoidismo. Corroborando com estes dados, Xiang et al. ${ }^{(3)}$ mostraram que 57 sujeitos com hipotireoidismo subclínico, que realizaram treinamento de exercício aeróbio moderado por seis meses, apresentaram melhora de $31 \%$ no fluxo sanguíneo muscular.

\section{- Função endotelial}

Dos mecanismos implicados no aumento da resistência vascular periférica dos hipotireoideos, a função endotelial exerce um papel em particular, devido a presença de receptores T3 e T4 na parece vascular. ${ }^{(4)}$ Investigações apontam que os níveis 
elevados de TSH também podem prejudicar a função endotelial. ${ }^{(45)}$ Em hipotireoideos subclínicos, Lekakis et al. ${ }^{(8)}$ demonstraram uma correlação negativa entre esta doença e a vasodilatação dependente do endotélio. Taddei et al. ${ }^{(22)}$ corroboram com estes dados, além de apontarem que em hipotireoideos subclínicos há também reduzida biodisponibilidade do óxido nítrico, potente vasodilatador liberado pelo endotélio, e que após terapia de reposição hormonal com levotiroxina, há aumento significante da vasodilatação.

A relação do hipotireoidismo com a disfunção endotelial parece ter origem com um perfil lipídico aterogênico, caracterizado pelo aumento do colesterol total e lipoproteínas de baixa densidade (LDL) oxidadas. ${ }^{(46)}$ Foi observado que a reposição de hormônios tireoideanos promove benefícios como a redução do colesterol total, do colesterol LDL e melhora na vasodilatação mediada pelo fluxo. ${ }^{(22)} \mathrm{No}$ entanto, é difícil conjecturar se a disfunção endotelial observada em hipotireoideos se deve ao é por efeito direto dos hormônios tireoidianos ou é consequência do perfil lipídico alterado. ${ }^{(47)}$ Sabe-se que a alta concentração de LDL está implicada em uma maior concentração de dimetilarginina assimétrica, um análogo natural da enzima óxido nítrico sintase endotelial, que inibe a produção de óxido nítrico, o que provoca uma redução na vasodilatação. ${ }^{(48)}$

O exercício físico tem demonstrado efeitos benéficos na disfunção endotelial, de modo a diminuir o risco aterogênico, melhorar o perfil lipídico e o fluxo sanguíneo em sujeitos normais ou com outras patologias como a hipertensão, diabetes e doenças cardiovasculares. ${ }^{(49-50)}$ Com pacientes acometidos por hipotireoidismo existe um único estudo, de Xiang et al. ${ }^{(3)}$, que observou que seis meses de treinamento físico em sujeitos com hipotireoidismo subclínico resultou em diminuição de $20 \%$ no colesterol total, $29 \%$ no LDL e 47,6\% dos triglicerídeos. Estas alterações foram acompanhadas por restauração da função endotelial avaliada pela melhora de $31,3 \%$ no fluxo sanguíneo e $61,5 \%$ na proteína $\mathrm{C}$ reativa.

\section{- Estresse oxidativo}

$\mathrm{Na}$ presença do estado hipotireoideo, a condição oxidativa parece estar elevada, facilitando a produção de radicais livres. ${ }^{(23-24,51)}$ Moulakakis et al. ${ }^{(23)}$ demonstraram que aortas de ratos com hipotireoidismo subclínico induzido apresentavam níveis de malondialdeído (produto final da peroxidação lipídica) e 8-oxoguanina-DNA (uma das bases modificadas no ácido desoxirribonucléico pela reação a espécies reativas de oxigênio) elevados em comparação a um grupo controle. Em humanos também já foi demonstrado que concentrações séricas de malondialdeído estavam significativamente aumentadas tanto em hipotireoideos subclínicos quanto clínicos. ${ }^{(24)}$

Embora estudos mostrem que o exercício físico é capaz de retardar a formação de radicais livres, aumentar a produção de antioxidantes endógenos e reduzir o estresse oxidativo nas doenças cardiovasculares como a hipertensão, diabetes e aterosclerose ${ }^{(52-53)}$, dados analisando seus efeitos sobre o estresse oxidativo em sujeitos com hipotireoidismo não foram encontrados mediante os critérios adotados para a seleção dos manuscritos.

\section{- Função autonômica}

Tem sido observada uma correlação entre níveis de TSH e aumento da atividade nervosa autonômica cardíaca simpática em hipotireoideos subclínicos. ${ }^{(12)}$ Este aumento está associado com os níveis das catecolaminas plasmáticas e índice de 
dispersão do segmento QT. ${ }^{(11,25,54-55)}$ Akcakoyun et al. ${ }^{(10)}$ investigaram a influência do hipotireoidismo subclínico na resposta ao exercício físico em termos de recuperação da frequência cardíaca e competência cronotrópica. Eles observaram que sujeitos acometidos por esta doença se mostram significativamente deficientes quanto às variáveis estudadas. Este fato sugere um comprometimento da reativação do sistema nervoso parassimpático no restabelecimento do balanço simpatovagal desses indivíduos após a realização do exercício físico.

Em hipotireoideos clínicos, Celik et al. ${ }^{(25)}$ indicam que esses sujeitos têm maior variabilidade da frequência cardíaca e após seis meses de terapia com reposição hormonal de levotiroxina esta disfunção parece ser revertida. Cacciatori et al. ${ }^{(26)}$ ao investigarem o balanço simpatovagal de hipotireoideos clínicos em resposta aos testes de sentar e levantar (protocolo adotado para verificar diferenças na atividade nervosa autonômica entre as posições sentado e em pé), verificaram que o componente de baixa frequência, correspondente a atividade nervosa simpática, encontrava-se significativamente elevado nos sujeitos hipotireoideos, quando se colocavam de pé.

Apesar do exercício físico não ter reduzido o componente de baixa frequência dos hipotireoideos subclínicos ${ }^{(10)}$, investigações conduzidas com hipertensos, diabéticos e obesos têm demonstrado redução significante tanto deste componente da variabilidade da frequência cardíaca quanto elevação do componente de alta frequência. ${ }^{(16-17)}$ Apesar destas evidências, os dados apresentados pelos estudos encontrados ainda são primários e insuficientes para determinar o real efeito do exercício físico aeróbio na atividade nervosa autonômica sugerindo a necessidade de novos estudos.

\section{- Pressão Arterial}

Os hormônios tireoidianos e tireoestimulante podem estar associados à elevação dos valores de pressão arterial sistólicos e diastólicos. ${ }^{(6,56)}$ Luboshitzky et al. ${ }^{(57)}$ verificaram que $20 \%$ de mulheres acometidas por hipotireoidismo subclínico eram hipertensas e possuíam pressão arterial média significativamente maior do que um grupo controle

Por outro lado, essa diferença de pressão arterial entre hipotireoideos e eutireoideos parece não ser observada durante o exercício físico. ${ }^{(3,31,58)}$ Arem et al. ${ }^{(58)}$ relataram não haver diferença entre o estado de hipotireoidismo subclínico e eutireoide para a pressão arterial sistólica e diastólica durante a realização de teste incremental na bicicleta ergométrica. Em outro estudo, observou-se que a pressão arterial e a frequência cardíaca não foram diferentes em pacientes com hipotireoidismo subclínico comparados aos indivíduos saudáveis durante teste incremental quando o ergômetro foi uma esteira. ${ }^{(31)}$

Em um único estudo, os hipotireoideos responderam diferentemente de eutireoideos, Mainenti et al. ${ }^{(31)}$ verificaram diferença significativa entre os níveis de pressão arterial de recuperação de hipotireoideas subclínicas quando comparados com os valores obtidos por eutireoideas num protocolo experimental até a fadiga. A magnitude de redução da pressão arterial foi quantificada durante o período de recuperação pós teste, sendo de apenas $0,71 \mathrm{mmHg}$ nas hipotireoideas, enquanto que as eutireoideas reduziram 5,33 $\mathrm{mmHg}$. Segundo os autores, é importante atentar para a pressão arterial diastólica de recuperação observada no primeiro e terceiro minutos pós-teste, pois, parece haver um comprometimento da função diastólica das hipotireoideas subclínicas, o que foi observado através de uma re- 
cuperação bastante lenta da pressão arterial diastólica entre o primeiro e terceiro minutos de recuperação

Apesar da resposta de pressão arterial desfavorável observada por Mainenti et al. ${ }^{(31)}$, em um protocolo de treinamento físico adotado por Xiang et $\mathrm{al}^{(3)}$ foi possível verificar redução significativa da pressão arterial média de $102 \pm 5$ para $97 \pm 7 \mathrm{mmHg}$. Entretanto, essa foi a única investigação conduzida em seres humanos que relatou redução da pressão arterial em sujeitos hipotireoideos. Claramente, é notada uma lacuna nos conhecimentos sobre como os hipotireoideos respondem, em termos de pressão arterial, a protocolos de exercícios físicos ou programas de treinamento. Nos poucos estudos existentes, estas respostas são observados em função de testes ergométricos. A validade externa destes dados é questionável pelo fato de que, na prática clínica, realizam-se exercícios contínuos ao invés de incrementais.

\section{Implicações práticas}

A prevalência de hipotireoidismo subclínico no mundo é de 4 a 10\%, sendo que de 3 a 18\% desses casos evoluem para a forma clínica. ${ }^{(2)}$ Esta prevalência é similar ao que ocorre para no diabetes melito. ${ }^{(59)}$ No entanto, apesar de existir um razoável número de investigações sobre a influência do exercício físico no diabetes, o efeito do exercício físico nas respostas cardiometabólicas de indivíduos com hipotireoidismo ainda é um tema pouco estudado, de modo que a capacidade dos hipotireoideos para realizar exercício físico é razoavelmente determinada, a influência do exercício físico sobre a função endotelial e atividade nervosa autonômica é pobremente esclarecida, e os efeitos do exercício físico sobre as demais complicações cardiovasculares promovidas por esta doença ainda não estão disponíveis na literatura. Ademais, o risco associado ao exercício físico também não foi determinado.

Instituições de saúde, como o American College of Sports Medicine ${ }^{(60-61)}$, recomendam a realização de um programa de exercício físico para diversos tipos de populações, incluindo indivíduos obesos, diabéticos, hipertensos e portadores de insuficiência cardíaca. Entretanto, devido à escassez de estudos, os dados encontrados até o momento sobre os efeitos do exercício físico no hipotireoidismo subclínico não permitem a formulação de recomendações para uma prescrição de exercício físico seguro e eficaz para os hipotireoideos.

Portanto, esta revisão chama a atenção do leitor para o fato de que o hipotireoidismo é uma enfermidade que afeta o sistema cardiovascular, mas não existem até o presente momento recomendações de como se prescrever exercício para esta população.

Os hipotireoideos podem apresentar disfunção ventricular compatível com insuficiência cardíaca. Este dado poderia estimular uma prescrição de exercício similar à recomendada pelas III Diretrizes Brasileiras de Insuficiência Cardíaca Crônica ${ }^{(62)}$ para pacientes com fração de ejeção ventricular comprometida. No entanto, a falta de dados na literatura torna esta perspectiva meramente especulativa, e, ao mesmo tempo, pressupõe caminhos teóricos a serem investigados para que futuramente se esclareça os riscos e benefícios para a prescrição de exercícios para os hipotireoideos. Para se alcançar este estado da arte, indica-se que os próximos estudos investiguem os efeitos agudos e crônicos dos exercícios físicos aeróbios e resistidos sobre as variáveis cardiometabólicas de humanos acometidos pelo hipotireoidismo. Estes estudos devem partir das informações prévias de que o exercício físico parece promover melhoria no fluxo sanguíneo e perfil lipídico, que pode levar a uma melhora na função endotelial em pacientes hipotireoideos. No entanto, 
possíveis benefícios do exercício físico sobre a modulação autonômica e estresse oxidativo ainda não estão estabelecidos.

Esta revisão revela claramente que o hipotireoidismo afeta as funções cardiovasculares por mecanismos diretamente envolvidos com receptores tireoideanos localizados no coração e vasos sanguíneos. A ação destes receptores não permite que benefícios nas funções cardiovasculares classicamente associados ao exercício físico em outras desordens cardiometabólicas possam ser extrapolados aos hipotireoideos. Portanto, esta revisão aponta para a necessidade de estudos sobre a capacidade e segurança do exercício físico e determinação da capacidade restauradora das funções cardiovasculares prejudicadas no hipotireoidismo.

\section{Agradecimentos/Financiamento}

Agradecemos aos autores dos estudos incluídos nesta revisão que contribuíram com suas informações. Salientamos ainda que a presente revisão não possui fonte de financiamento.

\section{Contribuição dos autores}

Alesandra Araújo de Souza contribuiu com a escrita, revisão da versão final e formatação do manuscrito para as normas da revista. Aluísio Henrique Rodrigues de Andrade Lima contribuiu com a escrita, formatação do manuscrito para as normas da revista e elaboração dos documentos necessários para a submissão. Thereza Sarmento da Nóbrega contribuiu com a escrita deste manuscrito. Alexandre Sérgio Silva orientou, contribuiu na elaboração, correção da escrita e revisão da versão submetida ao periódico.

\section{REFERÊNCIAS}

1. Biondi B, Klein I. Hypothyroidism as a risk factor for cardiovascular disease. Endocrine 2004;24:1-13.

2. McDermott MT, Ridgway EC. Subclinical hypothyroidism is mild thyroid failure and should be treated. J Clin Endocrinol Metab. 2001;86:4585-90.

3. Xiang GD, Pu J, Sun H, et al. Regular aerobic exercise training improves endotheliumdependent arterial dilation in patients with subclinical hypothyroidism. Eur J Endocrinol. 2009;161:755-61.

4. Klein I, Ojamaa K. Thyroid hormone and the cardiovascular system. N Engl J Med. 2001;344:501-9.

5. Mizuma H, Murakami M, Mori M. Thyroid hormone activation in human vascular smooth muscle cells: expression of type II iodothyronine deiodinase. Circ Res. 2001;88:313-8.

6. Saltiki K, Voidonikola P, Stamatelopoulos K et al. Association of thyroid function with arterial pressure in normotensive and hypertensive euthyroid individuals: A cross-sectional study. Thyroid Res. 2008;1:3.

7. Dagre AG, Lekakis JP, Papaioannou TG et al. Arterial stiffness is increased in subjects with hypothyroidism. Int J Cardiol. 2005;103:1-6.

8. Lekakis J, Papamichael C, Alevizaki M et al. Flow-mediated, endothelium-dependent vasodilation is impaired in subjects with hypothyroidism, borderline hypothyroidism, and high-normal serum thyrotropin (TSH) values. Thyroid. 1997;7:411-4.

9. Coria MJ, Pastran AI, Gimenez MS. Serum oxidative stress parameters of women with hypothyroidism. Acta Biomed. 2009;80:135-9.

10. Akcakoyun M, Emiroglu Y, Pala S et al. Heart rate recovery and chronotropic incompetence in patients with subclinical hypothyroidism. Pacing Clin Electrophysiol. 2010;33:2-5.

11. Galetta F, Franzoni F, Fallahi P et al. Heart rate variability and QT dispersion in patients with subclinical hypothyroidism. Biomed Pharmacother. 2006;60:425-30. 
12. Sahin I, Turan N, Kosar F, Taskapan C, Gunen H. Evaluation of autonomic activity in patients with subclinical hypothyroidism. J Endocrinol Invest. 2005;28:209-13.

13. Rondon MUPB, Brum PC. Exercício físico como tratamentonão-farmacológico da hipertensão arterial. Rev Bras Hipertens 2003;10:134-9.

14. Ciolac EG, Guimarães GV. Exercício físico e síndrome metabólica. Rev Bras Med Esporte 2004;10.

15. Arsa G, Lima L, Almeida SS et al. Diabetes Mellitus tipo 2: Aspectos fisiológicos, genéticos e formas de exercício físico para seu controle. Rev Bras Cineantropom Desempenho Hum 2009;11:103-11.

16. Edwards KM, Wilson KL, Sadja J, Ziegler MG, Mills PJ. Effects on blood pressure and autonomic nervous system function of a 12-week exercise or exercise plus DASH-diet intervention in individuals with elevated blood pressure. Acta Physiol (Oxf). 2011;203:343-50.

17. Goulopoulou S, Baynard T, Franklin RM et al. Exercise training improves cardiovascular autonomic modulation in response to glucose ingestion in obese adults with and without type 2 diabetes mellitus. Metabolism. 2010;59:901-10.

18. Kisso B, Patel A, Redetzke R, Gerdes AM. Effect of low thyroid function on cardiac structure and function in spontaneously hypertensive heart failure rats. J Card Fail. 2008;14:167-71.

19. Diekman MJM, Harms MPM, Endert E, Wieling W, Wiersinga WM. Endocrine factors related to changes in total peripheral vascular resistance after treatment of thyrotoxic and hypothyroid patients. European Journal of Endocrinology. 2001;144:339-46.

20. Gauna A, Messuti H, Papadopulos $G$ et al. Acute and chronic hypothyroidism are associated with similar left ventricular diastolic dysfunction relative to the euthyroid state: results of doppler echocardiographic comparisons. J Endocrinol Invest. 2011;34:e281-6.

21. Giannattasio C, Rivolta MR, Failla M et al. Large and medium sized artery abnormalities in untreated and treated hypothyroidism. Eur Heart J. 1997;18:1492-8.

22. Taddei S, Caraccio N, Virdis A et al. Impaired endothelium-dependent vasodilatation in subclinical hypothyroidism: beneficial effect of levothyroxine therapy. J Clin Endocrinol Metab. 2003;88:3731-7.

23. Moulakakis KG, Poulakou MV, Dosios T et al. Hypothyroidism and the aorta. evidence of increased oxidative DNA damage to the aorta of hypothyroid rats. In Vivo. 2008;22:603-8.

24. Torun AN, Kulaksizoglu S, Kulaksizoglu M et al. Serum total antioxidant status and lipid peroxidation marker malondialdehyde levels in overt and subclinical hypothyroidism. Clin Endocrinol (Oxf). 2009;70:469-74.

25. Celik A, Aytan P, Dursun $H$ et al. Heart rate variability and heart rate turbulence in hypothyroidism before and after treatment. Ann Noninvasive Electrocardiol. 2011;16:344-50.

26. Cacciatori V, Gemma ML, Bellavere F et al. Power spectral analysis of heart rate in hypothyroidism. Eur J Endocrinol. 2000 Sep;143(3):327-33.

27. Casimiro-Lopes G, Alves SB, Salerno VP et al. Maximum acute exercise tolerance in hyperthyroid and hypothyroid rats subjected to forced swimming. Horm Metab Res. 2008;40:276-80.

28. McAllister RM, Delp MD, Thayer KA, Laughlin MH. Muscle blood flow during exercise in sedentary and trained hypothyroid rats. Am J Physiol. 1995;269:H1949-54.

29. Bausch L, McAllister RM. Effects of hypothyroidism on the skeletal muscle blood flow response to contractions. J Vet Med A Physiol Pathol Clin Med. 2003;50:117-22.

30. Brenta G, Mutti LA, Schnitman M et al. Assessment of left ventricular diastolic function by radionuclide ventriculography at rest and exercise in subclinical hypothyroidism, and its response to L-thyroxine therapy. Am J Cardiol. 2003;91:1327-30.

31. Mainenti MRM, Teixeira PFS, Oliveira FP, Vaisman M. Impacto do Hipotireoidismo Subclínico na Resposta Cárdio-Pulmonar em Esforço e na Recuperação. Arq Bras Endocrinol Metab. 2007;51(9):1485-92.

32. Arem R, Escalante D. Subclinical hypothyroidism: epidemiology, diagnosis, and significance. Adv Intern Med. 1996;41:213-50.

33. Fazio S, Palmieri EA, Lombardi G, Biondi B. Effects of thyroid hormone on the cardiovascular system. Recent Prog Horm Res. 2004;59:31-50.

34. Klein I, Danzi S. Thyroid disease and the heart. Circulation. 2007;116:1725-35. 
35. Wiersinga WM. The role of thyroid hormone nuclear receptors in the heart: evidence from pharmacological approaches. Heart Fail Rev. 2010;15:121-4.

36. Ojamaa K, Klemperer JD, MacGilvray SS, Klein I, Samarel A. Thyroid hormone and hemodynamic regulation of beta-myosin heavy chain promoter in the heart. Endocrinology. 1996 Mar;137(3):802-8.

37. Mainenti MR, Teixeira PF, Oliveira FP, Vaisman M. Impact of subclinical hypothyroidism in cardiopulmonary response during effort and its recovery. Arq Bras Endocrinol Metabol. 2007;51:1485-92.

38. Tang YD, Kuzman JA, Said S et al. Low thyroid function leads to cardiac atrophy with chamber dilatation, impaired myocardial blood flow, loss of arterioles, and severe systolic dysfunction. Circulation. 2005;112:3122-30.

39. Niafar M, Toufan M, Ghafoori S, Aghamohammadzadeh N. Subclinical hypothyroidism effects on cardiac function. Pak J Biol Sci. 2009;12:1056-62.

40. Baumgartner-Parzer SM, Wagner L, Reining G et al. Increase by tri-iodothyronine of endothelin-1, fibronectin and von Willebrand factor in cultured endothelial cells.J Endocrinol. 1997;154:231-9.

41. Isumi Y, Shoji H, Sugo S et al. Regulation of adrenomedullin production in rat endothelial cells. Endocrinology. 1998;139:838-46.

42. Ojamaa K, Klemperer JD, Klein I. Acute effects of thyroid hormone on vascular smooth muscle. Thyroid. 1996;6:505-12.

43. Napoli R, Guardasole V, Zarra E et al. Impaired endothelial- and nonendothelial-mediated vasodilation in patients with acute or chronic hypothyroidism. Clin Endocrinol (Oxf). 2010;72:107-11.

44. Lansink M, Koolwijk P, van Hinsbergh V, Kooistra T. Effect of steroid hormones and retinoids on the formation of capillary-like tubular structures of human microvascular endothelial cells in fibrin matrices is related to urokinase expression. Blood. 1998;92:927-38.

45. Sellitti DF, Dennison D, Akamizu T et al. Thyrotropin regulation of cyclic adenosine monophosphate production in human coronary artery smooth muscle cells. Thyroid. 2000;10:219-25.

46. Duntas LH. Thyroid disease and lipids. Thyroid. 2002;12:287-93.

47. Giannotti G, Landmesser U. Endothelial dysfunction as an early sign of atherosclerosis. Herz. 2007;32:568-72.

48. Nishiyama Y, Ueda M, Katsura K et al. Asymmetric dimethylarginine (ADMA) as a possible risk marker for ischemic stroke. J Neurol Sci. 2010;290:12-5.

49. Zaros PR, Pires CE, Bacci M Jr., Moraes C, Zanesco A. Effect of 6-months of physical exercise on the nitrate/nitrite levels in hypertensive postmenopausal women. BMC Womens Health. 2009;9:17.

50. Ziegler S, Schaller G, Mittermayer F et al. Exercise training improves low-density lipoprotein oxidability in untrained subjects with coronary artery disease. Arch Phys Med Rehabil. 2006;87:265-9.

51. Messarah M, Boumendjel A, Chouabia A et al. Influence of thyroid dysfunction on liver lipid peroxidation and antioxidant status in experimental rats. Exp Toxicol Pathol. 2010;62:301-10.

52. Kimura H, Kon N, Furukawa $\mathrm{S}$ et al. Effect of endurance exercise training on oxidative stress in spontaneously hypertensive rats (SHR) after emergence of hypertension. Clin Exp Hypertens. 2010;32(7):407-15.

53. Lee S, Park Y, Zuidema MY, Hannink M, Zhang C. Effects of interventions on oxidative stress and inflammation of cardiovascular diseases. World J Cardiol. 2011;3:18-24.

54. Inukai T, Takanashi K, Kobayashi $\mathrm{H}$ et al. Power spectral analysis of variations in heart rate in patients with hyperthyroidism or hypothyroidism. Horm Metab Res. 1998;30:531-5.

55. Karthik S, Pal GK, Nanda N et al. Sympathovagal imbalance in thyroid dysfunctions in females: correlation with thyroid profile, heart rate and blood pressure. Indian J Physiol Pharmacol. 2009;53:243-52.

56. Menof P, Rand BC. A new treatment for polyneuritis. S Afr Med J. 1950;24:715-7.

57. Luboshitzky R, Aviv A, Herer P, Lavie L. Risk factors for cardiovascular disease in women with subclinical hypothyroidism. Thyroid. 2002;12:421-5. 
58. Arem R, Rokey R, Kiefe C, Escalante DA, Rodriguez A. Cardiac systolic and diastolic function at rest and exercise in subclinical hypothyroidism: effect of thyroid hormone therapy. Thyroid. 1996;6:397-402.

59. Shaw JE, Sicree RA, Zimmet PZ. Global estimates of the prevalence of diabetes for 2010 and 2030. Diabetes Res Clin Pract. 2010;87:4-14.

60. Medicine. ACotS. Appropriate Intervention Strategies for Weight Loss and Prevention of Weight Regain for Adults. Medicine and Science in Sports and Exercise. 2001.

61. Medicine ACotS. Exercise and Hypertension. 2004.

62. Bocchi EA M-BF, Ayub-Ferreira SM, Rohde LE, Oliveira WA, Almeida, Draisma A. III Diretrizes Brasileiras de Insuficiência Cardíaca Crônica. Prescrição do Exercício: Sociedade Brasileira de Cardiologia 2009;21-2.

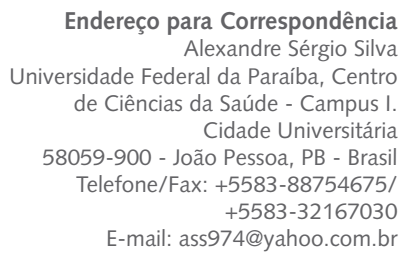

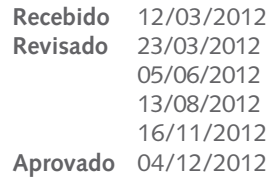

